Eric Fuß (Mannheim)/Marek Konopka (Mannheim)/ Angelika Wöllstein (Mannheim)

\title{
Perspektiven auf syntaktische Variation ${ }^{1}$
}

Abstract: In diesem Beitrag werden zunächst zwei Perspektiven auf sprachliche Variabilität diskutiert: Im Fokus stehen zum einen die Variation der Form und zum anderen die Variation der Funktion. Im Anschluss daran werden im Bereich der formalen Variation zwei Fälle eingehender untersucht: die AcI-Konstruktion mit ihren Kovarianten und die Relativsatzeinleitung mittels das oder was. Dabei wird der zuvor modellhaft entworfene methodische Rahmen auf die differenzierte Praxis linguistischer Forschung angewendet und das heuristische Potenzial des Prinzips der „Variationsreduktion“ genauer illustriert.

\section{Syntaktische Variabilität und Methodik ihrer Untersuchung}

\subsection{Variation zwischen formal unterschiedenen Varianten}

Von Variation wird in der Linguistik vor allem dann gesprochen, wenn die Konkurrenz zwischen zwei oder mehr sprachlichen Phänomenen gemeint ist, die als Varianten betrachtet werden. ${ }^{2}$ In der Regel lässt sich die von den Varianten konstituierte Variable ${ }^{3}$ funktional (semantisch, pragmatisch, grammatisch) beschreiben, wohingegen bei den Varianten deren formale Unterschiede im Vordergrund stehen. Zur Dominanz genau dieses Blickwinkels auf sprachliche Variabilität trugen entscheidend die Untersuchungen Labovs bei, der seit den 1960er Jahren den Begriff, linguistische Variable‘ im entsprechenden Sinn verwendet (vgl. Labov

1 Wir danken Felix Bildhauer, Patrick Brandt, Sandra Hansen-Morath, Roman Schneider und Ulrich Waßner für hilfreiche Diskussion und Unterstützung bei der Extraktion und Analyse der Daten.

2 Daneben wird ,Variation' manchmal z.B. auf Schwankungen der Frequenz eines Phänomens in verschiedenen Sprachgebrauchsbereichen bezogen. Zu der entsprechenden, hier aus Platzgründen ausgelassenen Perspektive vgl. Biber/Jones (2009) zu „type B studies“ und „type C studies“ sowie Bubenhofer et al. (2014, S. 36 ff.).

3 Zum einschlägigen Variablenbegriff vgl. - allgemeiner - Bortz/Döring (2006, S. 2 ff.) und - auf Linguistik bezogen - Durrel (2008, S. 274). 
1966, S. 15 ff.; 2008, S. 7). Der Blickwinkel an sich ist jedoch viel älter und begegnet uns explizit schon bei von der Gabelentz (1995, S. 84 ff.; dazu Kutscher 2014, S. 5 f.). Dort wird er von der althergebrachten lexikografischen Perspektive abgeleitet, die wir als die onomasiologische kennen. ${ }^{4}$ Analog wird heute zuweilen auch in der Grammatik von einer onomasiologischen Perspektive gesprochen. ${ }^{5}$ Sie fokussiert formale Variation, wie in Abbildung 1 am Beispiel der Variable ,konzessiver Konnektor' illustriert.

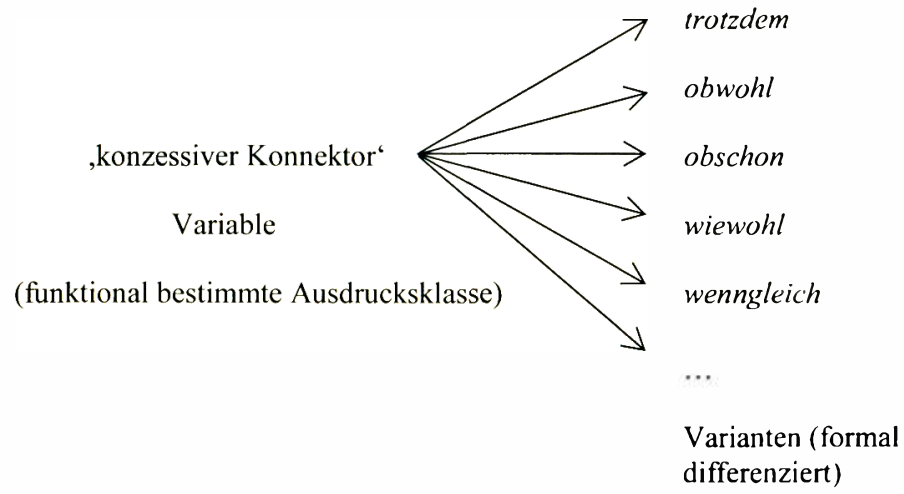

Abb. 1: Onomasiologische Perspektive und formale Variation ${ }^{6}$

Bei dem Beispiel handelt es sich um eine lexikalische Variation, die syntaktisch relevant ist. Die Konnektoren (zur genauen Definition vgl. Breindl/Volodina/Waßner 2014, S. 15f.) dienen nämlich dazu, semantische Relationen zwischen Propositionen explizit zu machen, und begründen somit komplexe syntaktische Strukturen. Verschiedene Konnektoren - selbst innerhalb einer semantischen Klasse - können dabei systematisch zu unterschiedlichen syntaktischen Strukturen ${ }^{7}$ führen, vgl. z.B.:

Es regnet in Strömen. Trotzdem nimmt sie das Fahrrad.

(2) Obwohl es in Strömen regnet, nimmt sie das Fahrrad.

4 So spricht Ammon (2005, S. 30) auch von „onomasiologischen Variablen“.

5 Vgl. z.B. Kutscher (2014, S. 5 ff.); Lehmann (2004, S. 149 ff.).

6 Die Reihe der Varianten ist natürlich durch die zahlreichen weiteren konzessiven Konnektoren erweiterbar, vgl. die Liste in Breindl/Volodina/Waßner (2014, S. 903 f.).

7 Die Unterschiede werden in 1.2.1 thematisiert. 
Die lexikalische Variation involviert hier also syntaktische Variation (mit der Variable ,konzessive Satzverknüpfung'), weil die Variable ,konzessiver Konnektor‘ die syntaktischen Einzelheiten nicht spezifiziert. Zwei weitere Fälle der bisher in der Forschung im Vordergrund stehenden formalen Variation werden in Abschnitt 2 und 3 genauer untersucht.

\subsubsection{Diagnose und Exploration der Variation}

Am Anfang einer Variationsbetrachtung steht meist die Feststellung, dass zwei oder mehr Phänomene Varianten voneinander sind. Um die Variable richtig zu charakterisieren, muss nach dem Tertium Comparationis des Variantenvergleichs gesucht werden, d.h. denjenigen Gemeinsamkeiten der Varianten, die ihre „Austauschbarkeit" ermöglichen. So ist etwa bei der Analyse gemeinsamer Kontexte von das und was als Relativsatzeinleiter die besondere morphologische Beschaffenheit des Bezugselements im Matrixsatz unübersehbar, vgl.:

Es ist $\left[_{\text {Neut. Sg. Nom. }}\right.$ das Neue, $\left[_{R S}\right.$ das/was den Anstoß gibt, eingefahrene Fahrwasser zu verlassen]].

Die vorläufige, bereits syntaktisch „angereicherte“ Beschreibung der interessierenden Variable lautet in diesem Fall: ,Relativpronomen zu einem Bezugselement im Neut. Sg.'.

Nach solchen noch weitgehend „impressionistischen“ Schritten der Variationsdiagnose und der ersten Variablendefinition kann mit der eigentlichen empirischen Exploration des Variationsphänomens begonnen werden. Diese soll hier primär in Bezug auf die korpusorientierte Herangehensweise thematisiert werden. Dabei wird angenommen, dass geeignete Korpusdaten bereits vorliegen und sich jetzt die Frage stellt, was die Variantenwahl beeinflusst. Zu überprüfen ist also die Wirkung von Faktoren, die als unabhängige Variablen einen Einfluss auf die interessierende, abhängige Variable nehmen könnten. Hier können sprachinterne und sprachexterne Einflussfaktoren unterschieden werden. $\mathrm{Zu}$ ersteren könnte in dem das/was-Beispiel etwa die Semantik des Bezugselements gehören - Faktoren dieser Art steuem die sog. Intrasprecher-Variation, können also prinzipiell bei ein und demselben Sprachbenutzer wirksam sein. Zu letzteren gehören Variablen wie Medium, Register, Textsorte, Erscheinungsjahr, Region etc., die sich auf soziolinguistische Variationsdimensionen wie Diachronie, Diatopie, Diastratie, Diaphasie, Diamesie abbilden lassen (solche Faktoren werden bei der AcI-Studie in Abschnitt 2 von Bedeutung sein). Bei ihnen tritt verstärkt die Intersprecher-Variation hervor, d.h., die Varianten können sich in Abhängigkeit von der Faktorausprägung auf verschiedene Sprachbenutzer verteilen. 


\subsubsection{Interpretation der Ergebnisse}

Werden sprachinterne Einflussfaktoren ermittelt, bei denen eine Faktorausprägung tendenziell ausnahmslos an eine Variante der abhängigen Variable gekoppelt ist, bedeutet dies, dass die sprachlichen Kontexte der Varianten - anders als ursprünglich angenommen - doch nicht gleich sind. In diesem Fall handelt es sich um sog. kombinatorische Varianten, was letztlich heißt, dass bei genauerer Bestimmung des sprachlichen Kontextes und bei der notwendig gewordenen Neudefinition der linguistisch relevanten Variable die Fälle, die bisher als Varianten angesehen worden waren, nicht mehr als Varianten zu betrachten sind. An dieser Stelle lässt sich der Exploration der Variation also ein Beitrag zur klassischen (nicht variationsorientierten) Linguistik abgewinnen, zumindest wenn man deren Hauptaufgabe wie Labov darin sieht, „[...] to eliminate variation by discovering the exact conditions that produce one variant or the other on the surface“ (2008, S. 6).

Für den Modellfall ,Relativsatzeinleitung mittels das oder was` kristallisiert sich ein sehr starker sprachinterner Einflussfaktor in ,Präsenz/Absenz eines lexikalischen Bezugsnomens` heraus. Die Erkenntnis, dass ein lexikalisches Bezugsnomen, wie es in das Pferd, das/ ${ }^{\star}$ was vorliegt, zumindest standardsprachlich die Wiederaufnahme mit was und somit die Variation ausschließt, muss jetzt bei der Neubestimmung der für die weitere Untersuchung interessanten Variable berücksichtigt werden (Genaueres in Abschnitt 3).

Werden sprachintern keine Variationsfaktoren ermittelt, die derart eindeutige Effekte zeigen, kann aus grammatischer Perspektive unter Umständen von freier Variation gesprochen werden. Oft werden aber schwächere Faktoren (auch deren Kombinationen) gefunden, die die Häufigkeit der Wahl einer Variante doch spürbar beeinflussen und damit die Annahme einer gänzlich freien Variation problematisch machen. Hier helfen inferenzstatistische Analysen und Messungen der Effektstärke, die gegebene Variation zwischen freier Variation und kombinatorisch bedingter Wahl zu verorten. Um das Zusammenwirken (die Interaktionen) verschiedener Faktoren einzuschätzen, sind komplexere statistische Verfahren wie Regressions- und/oder Varianzanalysen u.ä. hinzuzuziehen.

Davon unbeeinflusst erweist sich nicht nur bei echten kombinatorischen Varianten die sukzessive Einschränkung der Varianten und die Anpassung der zu untersuchenden abhängigen Variable als fruchtbar. Die systematische „Variationsreduktion“ hilft auch sonst, neue Einflussfaktoren zu entdecken und in ihrer Wirkung zu isolieren. Die Hauptaufgabe der traditionellen Linguistik (im Sinne Labovs) kann somit in der variationsorientierten Linguistik in abgeschwächter Form als heuristisches Prinzip fortleben. An die Stelle des Anspruchs, die Variation ganz zu eliminieren, tritt nun ihre untersuchungsbedingte Einschränkung. Die Methodik besteht dementsprechend in den Schritten: 
a) einleitende Untersuchung der anvisierten Varianten und ihrer Kontexte,

b) explorative Eliminierung „unechter“/nicht einschlägiger Variationsfälle,

c) Präzisierung/Neubestimmung der zu erforschenden abhängigen Variable.

Die Schritte a) bis c) können wiederholt werden, bis - idealerweise - keine als frei anzusehende Variation übrig bleibt.

\subsection{Variation zwischen funktional unterschiedenen Varianten}

Weit weniger üblich als die Betrachtung formaler Variation ist in der variationsorientierten Linguistik die umgekehrte Perspektive, bei der eine formal definierte Einheit am Ausgangspunkt steht. Bei von der Gabelentz (1995, S. 84 ff.; vgl. auch Kutscher 2014, S. 6 f.) wird sie mit derjenigen lexikografischen Herangehensweise in Zusammenhang gebracht, die wir als die semasiologische bezeichnen. ${ }^{8}$ Diese funktionale Variation wird in Abbildung 2 veranschaulicht, und zwar ausgehend von der Form $<$ trotzdem $>,{ }^{9}$ wie sie in den Korpora der geschriebenen Sprache erscheint.

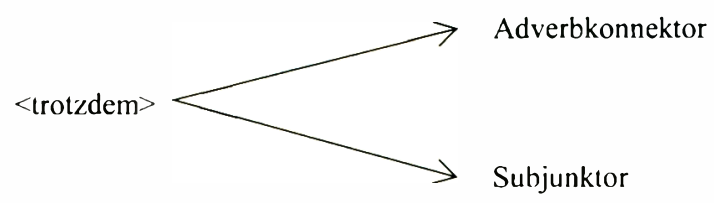

Variable

Varianten

(formal bestimmte Ausdrucksklasse)

(funktional differenziert)

Abb. 2: Semasiologische Perspektive und funktionale Variation

Wohlgemerkt, eine solche variationsorientierte Darstellung des Phänomens bleibt neutral gegenüber dessen theorieabhängigen Klassifizierungen als ein Fall von Polysemie, Homonymie (Homographie) u.ä. Die Unterschiede zwischen den Varianten betreffen die Verwendungsweise der Form $\langle$ trotzdem $>$.

8 So spricht Ammon (2005, S. 30) auch von „semasiologischen Variablen“.

9 Die Möglichkeit der Initialgroßschreibung ist hier eingeschlossen. 


\subsubsection{Diagnose und Exploration der Variation}

Das Tertium Comparationis des Variantenvergleichs ist bei funktionaler Variation viel leichter zu bestimmen als bei formaler Variation, nämlich als die formal definierte Einheit mit einer nur ganz allgemein gefassten Funktion. Für das Beispiel aus Abbildung 2 ist es mit ,<trotzdem>als Konnektor' bereits umrissen. Aufwendiger zu beschreiben sind dagegen die funktionalen Varianten. In unserem Modellfall sollen aus Platzgründen nur zwei solche Varianten berücksichtigt werden:

a) trotzdem als Adverbkonnektor, der syntaktisch in den Teilsatz integriert ist, der für das Konsequens der zugrundeliegenden Bedingungsrelation steht, vgl.:

(4) Es regnet in Strömen. Trotzdem nimmt sie das Fahrrad.

b) trotzdem als Subjunktor, der bei dem Antezedens der zugrunde liegenden Bedingungsrelation erscheint, vgl.:

Trotzdem es in Strömen regnet, nimmt sie das Fahrrad. ${ }^{10}$

Wenn es um die Wirkung sprachinterner Einflussfaktoren der Variantenwahl geht, so postuliert die bisherige Forschung die Bedeutung der unabhängigen Variable ,Betonung ' (TROTZdem vs. trotzDEM, vgl. Breindl/Volodina/Waßner 2014, S. 99 f.). Die Überprüfung dieser Variable ist in geschriebenen Texten nicht möglich, sodass sie mithilfe anderer Arten empirischer Evidenz durchzuführen wäre.

\subsubsection{Interpretation der Ergebnisse}

Bestätigt eine solche Überprüfung die Vermutung, dass die Subjunktorfunktion sehr stark mit der Ultimabetonung korreliert, während die Adverbkonnektorfunktion problemlos mit beiden Betonungen vereinbar ist, verlagert sich das linguistische Interesse: Es ist jetzt zusätzlich auf die Formvariation beim Adverbkonnektor trotzdem ausgerichtet (TROTZdem vs. trotzDEM), während die Funktionsvariation auf trotzDEM reduziert erscheint. In letzterem Fall muss die linguistisch interessante Variable also neu definiert werden als ,Verwendungsweise von trotzDEM'. Sind hier im Weiteren keine relevanten sprachinternen Einflussfaktoren zu finden, kann aus grammatischer Sicht von freier Variation gesprochen werden. $\mathrm{Zu}$

10 Es hätte außerdem etwa die Variante mit dem desintegrierten Konnektor angeführt werden können wie in Es regnet in Strömen. Trotzdem: Sie nimmt das Fahrrad. 
prüfen wäre allerdings noch der Einfluss sprachexterner Faktoren, und zwar sowohl bei der festgestellten formalen als auch bei der funktionalen Variation. Vor allem in letzterem Bereich legt die bisherige Forschung die Bedeutung des Faktors ,Region“ nahe (vgl. z.B. Duden 2016, S. 596), dem hier nicht mehr nachgegangen werden kann.

Bevor diese modellhaften Überlegungen zur Methodik anhand ausführlicherer Fallstudien in Abschnitt 2 und 3 konkretisiert werden, sei noch auf einen Punkt aufmerksam gemacht: Dass in unserem Beispiel im Laufe der semasiologisch bestimmten Exploration der funktionalen Variation die formale Variation mit der onomasiologischen Perspektive wiederkehrt, ist kein Zufall. Vielmehr ist dies typisch für eine durch die Variationsbrille betriebene und immer weiter strebende Erkundung der Grammatik. Dabei geht es generell um Korrelierung immer spezifischer werdender sprachlicher Funktionen mit immer präziser gefassten sprachlichen Formen, was einen ständigen Wechsel der Perspektiven bedingt. Dies sei für den in 1.2 behandelten Ausschnitt der Grammatik durch Abbildung 3 illustriert.

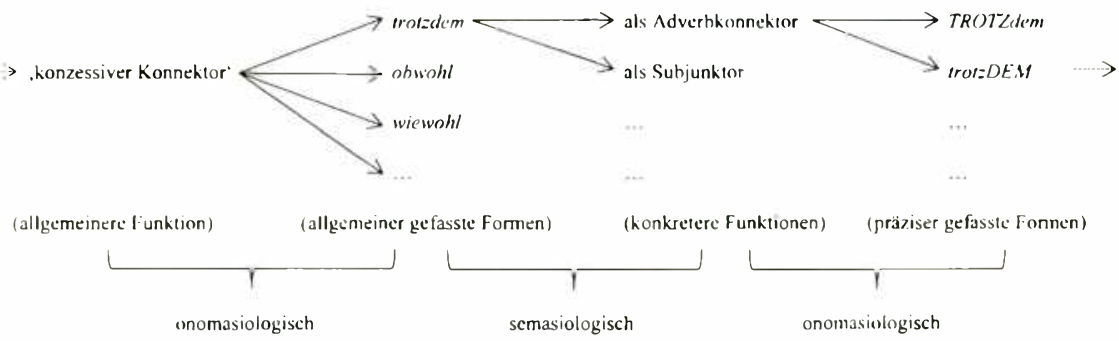

Abb. 3: Wechsel zwischen der onomasiologischen und der semasiologischen Perspektive am Beispiel der konzessiven Konnektoren

\section{Der Acl und seine finiten Kovarianten}

Komplementierungsmuster bei Verben, wie sie in sog. Satzbauplänen dargestellt zu finden sind (vgl. Duden 2016, RN 1444 ff.), stellen durch das Vorhandensein verschiedener Grundmuster für das einzelne Verb Variationsphänomene par excellence dar. Nicht nur im Hinblick auf nicht-sententiale Komplemente ist Variation vorzufinden. Die folgende Fallstudie dokumentiert in einem ersten, explorativen Teil quantitative Ergebnisse zur Verteilung von verschiedenen propositionsbezeichnenden Komplementen bei Wahrnehmungsverben und zur Abhängigkeit deren Häufigkeiten von sprachexternen Faktoren (Abschnitt 2.1). Die Vari- 
ation besteht in der syntaktischen Variabilität der Komplementierungsmuster und wird als Schwankung zwischen Realisierungsformen (Varianten) einer funktional definierten Variable gefasst.

Im Deutschen können Wahrnehmungsverben unterschiedliche Komplementierungsmuster aufweisen, realisiert z.B. als AcI-Struktur, dass-, wie- oder V2-Satz. In einem zweiten Untersuchungsteil (Abschnitt 2.2 und 2.3) wird gezeigt, dass nach einer Bestimmung der Varianten der Variable ,propositionale Komplementierung unter Rückbezug auf Forschungshypothesen eine schrittweise Neubestimmung der für die weitere Forschung relevanten abhängigen Variable nötig ist.

\subsection{Ein Blick in die Korpora}

Im gegenwärtigen Deutsch kommen obligatorisch $z u$-lose Infinitivkomplemente mit Wahrnehmungs-/Perzeptionsverben wie sehen, hören, fühlen, spüren vor, vgl. (6) und (7):

Klaus meinte, er habe den Kerl jedes Mal, wenn er ging, im Hausflur rumschleichen sehen. (DIV/SFM.00001, 2008)

Die Japaner schreien nicht vor dem Spiel, nur während des Spiels, ihr Schreien ist oft eine Art Quieken, auch ein staunendes Raunen, und alles zusammen klingt, als stünde man am Strand und hörte die Wellen rauschen, die Möwen und den Wind. (U02/JUN.01239, 2002)

Diese Verben bilden gemeinsam mit dem Infinitiv die AcI-Konstruktion, ${ }^{11}$ treten aber auch mit anderen Komplementierungsmustern auf. Um einen Eindruck von der Variation der Komplementierungsmuster zu bekommen, haben wir je ca. 500 Belege für die Verben sehen und hören jeweils aus DEREKo ${ }^{12}$ (Printme-

11 Auf die Struktur in (6) und (7) wird mit unterschiedlichen Termini verwiesen: Exceptional Case Marking (ECM), Raising-to-Object Construction oder - der klassischen lateinischen Grammatiktradition folgend - accusativus cum infinitivo (AcI), vgl. Zifonun/Hoffmann/Strecker (1997), Eisenberg (2013), Duden (2016). Nicht betrachtet werden hier $z u$-lose Infinitivkomplemente mit kausativen bzw. permissiven Verben wie lassen, heißen, machen, eingeschränkt produktive Konstruktionen mit wissen, meinen, finden, haben und im Weiteren: Infinitive mit (i) Modalverben, (ii) Bewegungsverben in Finalkonstruktionen und (iii) Verben wie bleiben, finden, haben, legen sowie (nicht) brauchen, helfen, lehren, lernen und (nicht kausativem) heißen, vgl. u.v.a. Reis (1976, 2001), Zifonun/Hoffmann/Strecker (1997, S. 1411), Duden (2016, RN 577).

$12 \mathrm{Vgl}$. www.ids-mannheim.de/kl/projekte/korpora/. 
dien) und DECOW14 ${ }^{13}$ (Web) manuell annotiert (insgesamt ca. 1.970 Belege). Eine erste Hypothese war, dass es sich beim AcI um ein eher standardsprachliches Phänomen handelt. Dies könnte sich dann in Verteilungsunterschieden in den beiden Korpora niederschlagen, denn im Webkorpus DECOW14 sind verstärkt nichtstandardsprachliche Texte zu erwarten. Die Exploration der Stichproben hat Folgendes gezeigt: Insgesamt sind sententiale Komplementierungsmuster eher selten. Der Anteil der AcI-Komplementierung beträgt dabei ca. 3\% aller Komplementierungsmuster. Häufiger ist insbesondere die nicht-sententiale Komplementierung durch eine NP mit einem Anteil von insgesamt ca. 60\% (vgl. Abb. 4).
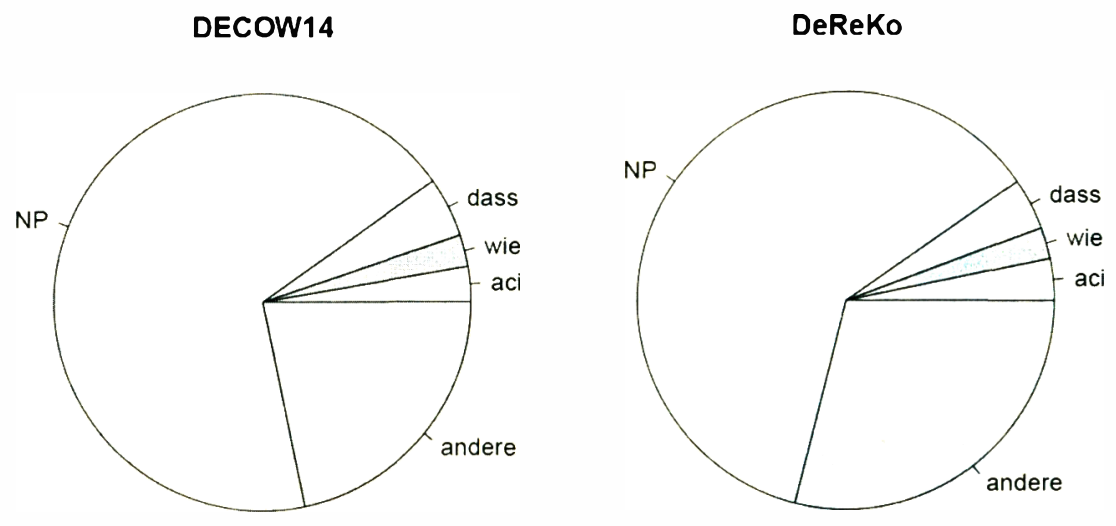

Abb. 4: Komplementtypen in DECOW14 und DEREKo

Das Komplementierungsverhalten variiert für propositionale Komplementierungen mit dass-, wie- über AcI- bis zu V2-Strukturen (die in der Abbildung in „andere“ enthalten sind) von DECOW14 zu DEREKo und auch von Verb zu Verb nur unwesentlich. Die relative Häufigkeit der Komplementierungsmuster schwankt aber innerhalb der DeREKo-Daten, und zwar in Bezug auf die Dimensionen ,Medium und ,Register ${ }^{14}$ Es fällt vor allem auf, dass die AcI-Strukturen in den Kategorien .Bücher' (Medium) und ,literarische Texte‘ (Register) überrepräsentiert sind. ${ }^{15}$

13 Vgl. Schäfer/Bildhauer (2012).

14 Die DeREKo-Daten wurden semi-manuell mit Metadaten zu ,Medium' und ,Register' angereichert, vgl. Bubenhofer et al. (2014).

15 Vgl. die Dokumentation der Ergebnisse unter http://hypermedia.ids-mannheim.de/call/public/ korpus.ansicht. 
Um diesem ersten Indiz auf die Abhängigkeit der AcI-Häufigkeit von sprachexternen Faktoren weiter nachzugehen, wurde zusätzlich eine größere Datensammlung ausgewertet. Darin wurden die in DeReKo enthaltenen AcIs vom Typ ,Infinitiv | Wahrnehmungsverb [| Form von haben] ${ }^{16}$ erfasst (nach Bereinigung 26.477 Belege), die im Korpus mit der Häufigkeit von 2,7 pro eine Million Wörter vorkommen. Mit Blick auf einbettende Verben ergibt sich hier eine Verteilung von $81,1 \%$ der AcIs bei sehen und 17,4\% bei hören, die restlichen Verben (fühlen, spüren) erscheinen marginal. Überprüft man die Verteilung der unter sehen eingebetteten AcIs in Abhängigkeit von ,Medium', bestätigt sich das bisherige Bild: In der Kategorie ,Bücher' ist der Anteil von sehen mit AcI an allen Vorkommen des Lemmas sehen etwa zweieinhalbmal höher als in der Kategorie „Presse“, die hier den zweiten Rang einnimmt (vgl. Abb. 5).

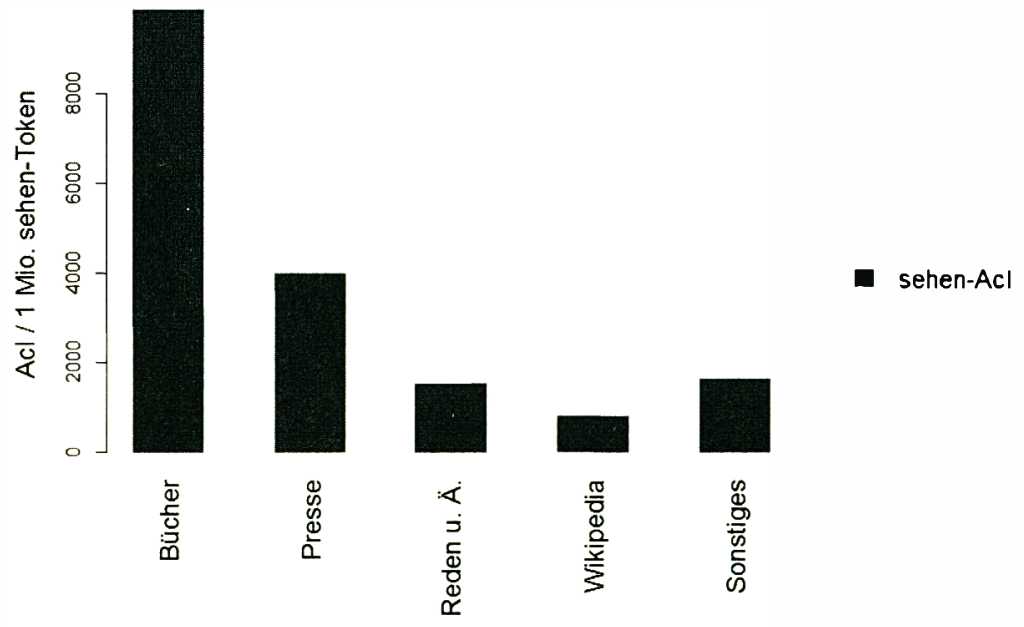

Abb. 5: sehen-Acl nach ,Medium ' in DEREKo ${ }^{17}$

Außerdem zeichnet sich eine Abnahme des AcI in neuester Zeit ab: Der AcI-Anteil an allen sehen-Vorkommen fällt innerhalb der vergangenen drei Jahrzehnte um mehr als ein Drittel (vgl. Abb. 6).

16 Z.B.: Wir haben es schon kommen sehen oder ... als sie die leeren Busse fahren gesehen haben.

17 Ein Chi-Quadrat-Test mit aus der Anzahl aller sehen-Token abgeleiteten erwarteten Häufigkeiten ergibt: $\chi^{2}(4)=2673.38 ; p<2.2 e-16$. Die Pearson-Residuen für die abgetragenen Kategorien

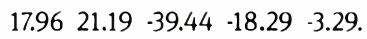




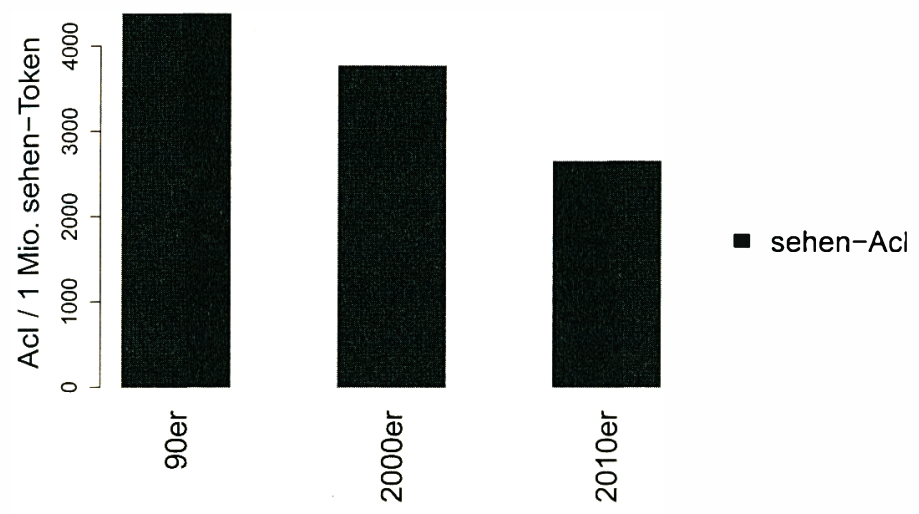

Abb. 6: sehen-Acl nach ,Zeit' in DEREKo ${ }^{18}$

Das Fazit aus dem explorativen Teil der Studie ist, dass die Korpusanalysen keine Hinweise etwa auf die Beschränkung bestimmter propositionaler Komplementierungen auf standardsprachliche Kontexte geben, wohl aber Unterschiede innerhalb des standardsprachlichen Gebrauchs selbst nahe legen: Die Indizien für eine Medium- und Registerabhängigkeit der AcI-Häufigkeit und für eine diachrone Entwicklung sind gegeben.

\subsection{Die Problematik der Strukturzuweisung zu-loser Infinitivkomplemente und die daraus resultierende Variablenbestimmung}

Unter einer Perspektive, die finite Komplementierungsmuster (im Folgenden beschränkt auf dass-, wie- und V2-Sätze) mit dem AcI zusammenzuführen versucht, besteht erstens die Problematik der Bildung einer gemeinsamen Variable und zweitens die einer entsprechend gültigen Festlegung auf mögliche Varianten finiter Strukturen zum AcI.

Die Problematik der Variablendefinition basiert auf der Strukturzuweisung des AcI mit bzw. relativ zu seinem einbettenden Verb. In der Literatur werden folgende Analysevorschläge diskutiert: a) Es handelt sich bei AcI plus einbetten-

18 Ein Chi-Quadrat-Test mit aus der Anzahl aller sehen-Token abgeleiteten erwarteten Häufigkeiten ergibt: $\chi^{2}(2)=810.51 ; p<2.2 \mathrm{e}-16$. Die Pearson-Residuen für die abgetragenen Kategorien $17.317 .57 \cdot 21.30$. 
des Verb um eine Verknüpfung zu einem Prädikatsausdruck (i.S.v. Bech 1955/1957) einer monoklausalen kohärenten Struktur mit Verbalkomplex, die aber in der Bikonstituenz des AcI resultiert (vgl. (8) mit den Bestandteilen des AcI verteilt auf VP und den Verbalkomplex), dazu u.a. Haider (1984), Zifonun/Hoffmann/ Strecker (1997, S. 1411), und b) um die Einbettung eines propositionsbezeichnenden Komplements und Monokonstituenz des AcI, dessen monoklausale Struktur mit dem einbettenden Verb (vgl. (9) mit vollständig eingebetteter Phrase XP) zumindest als kontrovers gelten kann, dazu u.a. Rosenbaum (1967), Reinhart/Reuland (1993), Eisenberg (1994), Zifonun/Hoffmann/Strecker (1997, S. 1422 f.).

$$
\begin{aligned}
& \text { Man konnte }\left[{ }_{\mathrm{VP}} \text { den Nachbarn die Treppe }\left[_{\mathrm{V} / \mathrm{VK}} \text { heraufsteigen hören }\right]\right] \text {. } \\
& \text { Man konnte }\left[_{\mathrm{VP}}\left[_{\mathrm{XP}} \text { den Nachbarn die Treppe heraufsteigen }\right]\right. \text { hören]. }
\end{aligned}
$$

Bis heute ist die Analyse des AcI Gegenstand kontroverser Diskussion in unterschiedlichen theoretischen Kontexten. ${ }^{19}$ Unter der Annahme von Bikonstituenz und Monoklausalität gelten die für Kohärenz üblichen Eigenschaften jedoch mit spezifischen Beschränkungen, die u.a. die Anbindungsreihenfolge des zentralen Akkusativs betreffen (Zifonun/Hoffmann/Strecker 1997). Zu den syntaktischen Besonderheiten zählen ein i.d.R. fehlendes Passiv beim AcI-Infinitiv sowie Besonderheiten bei der Reflexivierung, vgl. Reis (1976).

Unter der Annahme von Monokonstituenz und Biklausalität (wie in (9)) ist die Anaphorisierung als eine Einheit möglich, vgl. (10)-(11). Auch haben Schlussfolgerungsbeziehungen keine Geltung. So kann der zentrale Akkusativ nicht allein Gegenstand der Perzeption sein, vgl. (12).

$$
\begin{aligned}
& \text { man hört [ihn kommen] } \\
& \text { man hört [es] / *man hört es ihn/man hört ihn (genuin 2-stellig/Ana- } \\
& \text { lepse des Infinitivs) } \\
& \text { man hörte nicht den Nachbarn die Treppe hinaussteigen } \neq \text { man hörte } \\
& \text { den Nachbarn nicht }
\end{aligned}
$$

Demgegenüber unkontrovers bilden die anderen propositionsbezeichnenden Komplemente mit ihren einbettenden Verben immer eine komplexe biklausale Struktur.

19 Vgl. u.a. Reis (1976); Chomsky/Lasnik (1977); Mittwoch (1990); Bausewein (1991); Zimmermann (1991); Felser (1999); Sternefeld (2006); Haider (2010); Speyer (2015). 


\subsection{Variablendefinition und Variantenfestlegung}

Die Bestimmung des AcI als propositionsbezeichnendes Komplement findet seine Bestätigung in Umschreibungsmöglichkeiten des AcI durch finite Verbendstrukturen - eingeleitet mit dass und wie - oder durch V2-Sätze.

$$
\begin{aligned}
& \text { Man hört [den Nachbar die Treppe heraufsteigen]. } \\
& \text { Man hört [dass der Nachbar die Treppe heraufsteigt]. } \\
& \text { Man hört [wie der Nachbar die Treppe heraufsteigt]. } \\
& \text { Man hört [der Nachbar sei die Treppe heraufgestiegen]. }
\end{aligned}
$$

So können propositionsbezeichnende Komplemente, die von AcI-Verben eingebettet werden, unterschiedlichen syntaktischen Typs sein. Als eine Variante zum AcI erfüllen sie jedoch die Bedingung, als propositionsbezeichnendes Komplement eines Perzeptionsverbs auftreten zu können: Daraus lässt sich zunächst das folgende Funktion-Form-Korrelat bilden, das das propositionsbezeichnende Komplement des Wahrnehmungsverbs als Variable I festlegt:

\begin{tabular}{ll}
\hline Variable I & Varianten \\
\hline & Acl \\
\hline propositionsbezeichnendes Komplement & $\frac{\text { dass-Satz }}{\text { wie-Satz }}$ \\
& V2-Satz \\
\hline
\end{tabular}

Betrachtet man die Distribution der vorerst bestimmten Varianten genauer, wird jedoch ihre Einschränkung und die Neubestimmung der zu untersuchenden Variable nötig: Dass-Sätze weisen keine notwendige direkte Perzeption bzw. zeitliche Überlappung mit dem Matrixverb auf (vgl. (17)) und V2-Sätze sind nur unter Ausschluss direkter Perzeption/zeitlicher Überlappung möglich (vgl. (18) vs. (19)). Nur wie-Sätze sind wie AcIs auf direkte Perzeption/zeitliche Überlappung beschränkt (vgl. (20) und (21)).

(17) Ich höre, dass du dich beschwert hast.

(18) Ich höre, der Nachbar sei die Treppe heraufgestiegen.

(19) \#Ich höre, der Nachbar steigt die Treppe herauf.

(20) «Ich höre, wie du dich beschwert hast.

(21) *Ich höre den Nachbar die Treppe hinaufgestiegen sein. 
Dies führt zur eingeschränkten Variable II:

\begin{tabular}{ll}
\hline Variable II & Varianten \\
\hline $\begin{array}{l}\text { propositionsbezeichnendes Komplement, } \\
\text { direkte Perzeption }\end{array}$ & Acl \\
\hline
\end{tabular}

Übrigens kann auch die Doppelfunktion „propositionale Interpretation des Komplements (Monokonstituenz) und Strukturierung unter Bikonstituenz" nur eine wie-Struktur abbilden (vgl. (22); zur Problematik der wie-Komplemente u.a. Thurmair 2001). Nur beim wie-Satz kann eine Konstituente (unter pronominaler Wiederaufnahme) auch als Komplement des Wahrnehmungsverbs auftreten, vgl. (22b). Dass-Strukturen etwa erlauben das nicht, vgl. (23).

(22) a. Man hört, wie der Nachbar die Treppe heraufsteigt.

b. Man hört den Nachbar, wie er die Treppe heraufsteigt.

(23) a. Man hört, dass der Nachbar die Treppe heraufsteigt.

b. *Man hört den Nachbar, dass er die Treppe heraufsteigt.

Die wie-Sätze bringen jedoch die Problematik der Polysemie auf lexikalischer wie auf struktureller Ebene mit sich, vgl. Zimmermann (1991). Eine kleine Untersuchung zur Desambiguierung der wie-Sätze in einer DEREKo-Stichprobe von 250 Belegen wies nur $14 \%$ der wie-Sätze als Komplementsätze aus, bei $86 \%$ handelte es sich um wie-Fragen, wie-Graduierungen, wie-Adverbiale u.a. Unter den 35 wieKomplementen fanden sich auch zwölf Sätze, die kaum als AcI paraphrasierbar erscheinen, darunter vier im Passiv. Während also passivierte wie-Komplementsätze nicht unüblich sind, vgl. (24), ist Passiv des AcI-Infinitivs extrem selten, vgl. Belege (25) und (26) aus DECOW14. ${ }^{20}$

(24) Am schönsten finde ich an meinem Beruf, dass ich sehe, wie die Kinder lieben und geliebt werden.

(25) Sie sah des Gartens Früchte reifen und gepflückt werden.

(26) Denn so viele wir täglich sterben sehen, so viele sehen wir geboren werden.

20 Im Übrigen scheinen auch passivierte Perzeptionsverben mit propositionalem Komplement (z.B. er wurde schreien gehört) selten zu sein. 
Die Verarbeitungsprobleme, die der AcI bereits in der Aktivstruktur mit sich bringt, scheinen sich durch das Passiv noch zu potenzieren; offenbar handelt es sich um eine stark markierte Konstruktionsvariante; eine Ausweichmöglichkeit kann hier der wie-Satz sein, der die Struktur entzerrt, indem er die beiden Propositionen auf zwei (finite) Sätze verteilt und darüber hinaus den schwer zu verarbeitenden Verbkomplex vermeidet. Regulär alternieren kann somit der AcI nur mit dem wieSatz im Aktiv. Für die Variablenbestimmung führt das zur folgenden weiteren Präzisierung:

\begin{tabular}{ll}
\hline Variable III & Varianten \\
\hline $\begin{array}{l}\text { propositionsbezeichnendes Komplement, } \\
\text { direkte Perzeption, im Aktiv }\end{array}$ & $\mathrm{Acl}$ \\
\hline
\end{tabular}

In künftigen Untersuchungen muss eine Überprüfung von Einflussfaktoren für die Auswahl der beiden Varianten an größeren Datenmengen erfolgen. Dabei dürften qualitative und quantitative Untersuchungen zu den wie-Komplementsätzen mit einem Akkusativ, der im wie-Satz pronominal wiederaufgenommen wird (vgl. (22b)), eine besondere Rolle spielen. ${ }^{21}$

\section{Varianten der Relativsatzeinleitung}

\subsection{Einleitung}

Im Standarddeutschen werden attributive Relativsätze üblicherweise durch ein so genanntes $d$-Pronomen eingeleitet, das in verschiedenen Kasus stehen kann und in Genus und Numerus mit dem Bezugselement des Relativsatzes kongruiert (der, die, das, dem etc.). Unter bestimmten Umständen kann allerdings neben der $d$-Form das auch das $w$-Pronomen was als Relativum auftreten:

(27) a. Alles, was die Zuschauer dort sehen, ist Lug und Trug. (Niederösterreichische Nachrichten, 17.1.2013, NÖN Großformat, Ressort: Meinungen; PRO \& KONTRA)

21 Vgl. Fortmann (2013). 
b. Das, was wir machen, ist das, was uns gefällt. (Braunschweiger Zeitung, 4.6.2007; Das, was wir machen, ist das, was uns gefällt)

c. Das Beste, was Microsoft heute tun kann, ist, Yahoo zu kaufen. (Hannoversche Allgemeine, 8.11.2008, S. 15; Microsoft lässt Yahoo abblitzen)

Als erste Variationsdiagnose können wir festhalten, dass ein Relativpronomen mit den Merkmalen Neutrum, Singular, Nominativ/Akkusativ (Variable) durch die Varianten das und was realisiert werden kann: ${ }^{22}$

\begin{tabular}{ll}
\hline Variable & Varianten \\
\hline Relativpronomen (Neut. Sing., Nom./Akk.) & $\frac{\text { das }}{\text { was }}$ \\
\hline
\end{tabular}

In deskriptiven Grammatiken werden das und was in der Regel allerdings als kombinatorische Varianten aufgefasst. Danach stellt das den Standardfall dar, während was als Ausnahme behandelt wird, die nach Indefinita (alles, vieles etc.), Demonstrativa (das, dasjenige etc.) und nominalisierten Adjektiven (das Gute/ Beste/Einzige etc.) erscheint (vgl. z.B. Duden 2009, S. 1031 f.). Aus dieser Perspektive liegt also gar keine echte Variation vor; vielmehr ist die Verteilung vollständig durch grammatische Regularien bestimmt. Bei näherer Betrachtung wird aber deutlich, dass sich die Elemente, die was auslösen, nicht einheitlich verhalten. So treten in Kombination mit substantivierten Adjektiven sowohl das als auch was als Relativum auf (vgl. auch Duden 2009, S. 1032):

Denn nicht das Gute, das ich will, tue ich, sondern das Böse, das ich nicht will, das führe ich aus. [...] Und dass es Kraft braucht, Mut, trotz diesem Widerstreit all das Gute, was wir zustande bringen, zu achten, zu verstärken. Und nicht verächtlich zu machen! (Die Südostschweiz, 18.2.2006; "Gutmensch» - ein Unwort)

Entsprechende Nominalisierungen sind mit beiden Einleitungstypen kompatibel und unterscheiden sich in dieser Hinsicht sowohl von Substantiven (die das verlangen) als auch von Determinierern/Indefinita (die was auslösen). In der Folge

22 Keine Beachtung findet hier die stilistisch markierte Variante der Relativsatzeinleitung durch einen morphologisch komplexen $w$-Determinierer (welch-es), da sie im Wesentlichen die gleiche Distribution wie $d$-Pronomina aufweist. 
soll ausgehend von einem Analyseansatz, der was nicht als Spezialfall, sondern als Default-Relativum betrachtet (Brandt/Fuß 2014, 2015) das Relativierungsverhalten substantivierter Adjektive, also der stärker variable Bereich, näher betrachtet werden. Dabei werden weitere Faktoren herausgearbeitet, welche die Variation zwischen verschiedenen Einleitungsformen steuern. Es wird gezeigt, dass die Wahl zwischen das und was wesentlich davon abhängt, ob eine gesteigerte Form des Adjektivs vorliegt: Substantivierte Superlative verlangen was als Relativum, während bei anderen Formen sowohl das als auch was auftreten kann - der Variationsraum ist also innerhalb der substantivierten Adjektive noch weiter einzuschränken. Darüber hinaus wird der Nachweis erbracht, dass die Wahl des Relativums zusätzlich von grammatikexternen Faktoren beeinflusst wird, wobei wir uns auf die Reflexe der Unterscheidung Mündlichkeit vs. Schriftlichkeit konzentrieren.

\section{2 was als Default-Relativum}

Ausgehend von der Beobachtung, dass die das/was-Alternation wesentlich abhängig ist von der Präsenz eines lexikalischen Bezugsnomens, entwickeln Brandt/ Fuß $(2014,2015)$ einen alternativen Beschreibungsansatz, der Ideen von Behaghel (1928) und Wiese (2013) aufgreift. Grundlage ist dabei die folgende empirische Generalisierung, die zum Ausdruck bringt, dass ein Substantiv im Neutrum Singular stets die $d$-Form das als Relativum verlangt:

$$
\begin{aligned}
& \text { Generalisierung: Relativierung mittels das } \\
& \mathrm{N}_{\text {[Neutrum Singular] }} \rightarrow \text { das }
\end{aligned}
$$

Der Effekt, den die Präsenz eines lexikalischen Bezugsnomens auf die Wahl des Relativums hat, lässt sich an einer Korpusstudie nachvollziehen, mithilfe derer Brandt/Fuß (2015) die relative Häufigkeit von das/was in Konstruktion mit Elementen wie Demonstrativa und Indefinita untersucht haben, die Relativierung durch was verlangen (vgl. (27)).

Wie die Befunde in Abbildung 7 zeigen, wird durch die Hinzufügung eines Substantivs auch bei Elementen wie alles, die für sich genommen was als Relativum verlangen, Relativierung mittels $d$-Pronomen quasi obligatorisch (vgl. Brandt/ Fuß 2015 für eine detaillierte Aufarbeitung der Ergebnisse). Um diesen starken Faktor zu modellieren, schlagen Brandt/Fuß $(2014,2015)$ eine Analyse vor, nach der die Wahl zwischen $d$ - und $w$-Relativum wesentlich abhängig ist von Eigenschaften des Bezugselements. Im Gegensatz zu traditionellen Ansätzen wird was aber nicht als Ausnahme betrachtet, sondern als unterspezifiziertes Element ana- 
lysiert, das verwendet wird, wenn die spezifischeren Einsetzungsbedingungen von das nicht erfüllt sind:

a. das erfordert die Präsenz eines lexikalischen Bezugsnomens;

b. In allen anderen Fällen wird was als Default-Relativum verwendet.

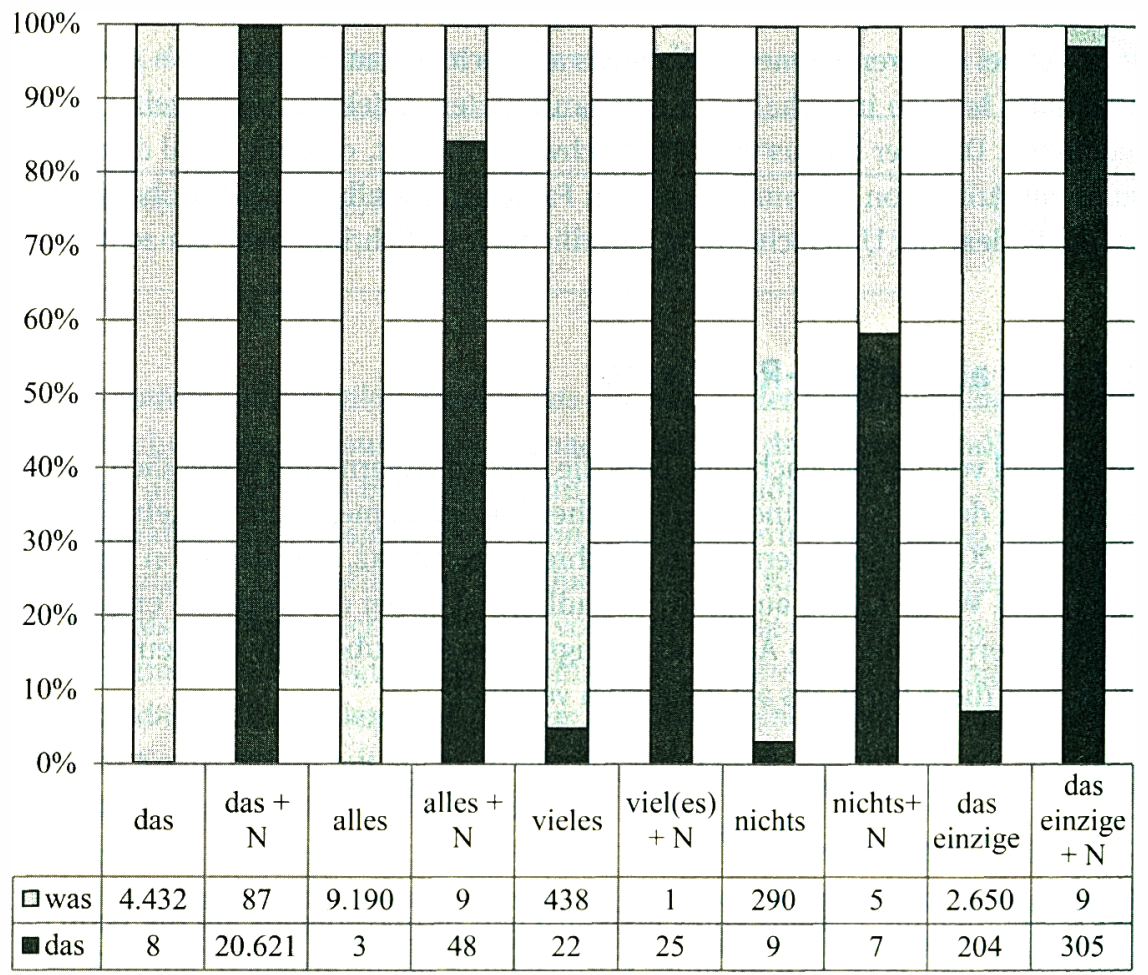

Abb. 7: Präsenz/Absenz von $\mathrm{N}$ bei Elementen, die was als Relativum verlangen (DEREKo, Recherche im Connexor-Teilarchiv, Juni 2014)

Dieser Beschreibungsansatz basiert auf der Idee, dass die Wahl zwischen das und was einer Spezifizitätsbedingung wie der Elsewhere Condition (Kiparsky 1973, 1982) unterliegt: Sind mehrere Formen mit einem Einsetzungskontext kompatibel, muss das spezifischste Element verwendet werden. Für diese Analyse spricht, dass sie eine einheitliche Beschreibung von verschiedenen Relativsatzkonstruktionen ermöglicht, die durch $w$-Pronomen eingeleitet werden und sich dadurch auszeichnen, dass es ihnen an einem substantivischen Bezugselement fehlt. Neben 
attributiven was-Relativsätzen betrifft dies freie Relativsätze, weiterführende Relativsätze (die sich auf ein Prädikat bzw. eine Proposition beziehen) sowie Relativsätze, die sich an Zitate bzw. zitatähnliche Ausdrücke anschließen, vgl. (31).

Hauptsache, die Stoffe sind flauschig weich und vermitteln ein Gefühl von «Wellness», was soviel bedeutet wie Wohlgefühl. (St. Galler Tagblatt, 17.9.1997; Ein Modewinter)

\subsection{Substantivierte Adjektive}

Die Analyse von Brandt/Fuß scheint zunächst ebenfalls nahe zu legen, dass die Alternation zwischen das und was gänzlich durch Regeln der Grammatik bestimmt ist. Wir haben aber bereits eingangs erwähnt, dass nach substantivierten Adjektiven beide Formen zulässig sind (vgl. auch Duden 2009, S. 1032). Eine nähere Betrachtung zeigt allerdings, dass die empirischen Verhältnisse komplexer sind und der Variationsbereich tatsächlich noch weiter eingeschränkt werden muss. Es müssen drei Haupttypen unterschieden werden (vgl. Brandt/Fuß 2015; Fuß i.Ersch.):

(32) a. elliptische Lesarten (getilgtes Substantiv):

ausschließlich das

b. substantivierte Superlative (das Beste, nicht-elliptisch): fast ausschließlich was

c. substantivierte Positive (das Gute, nicht-elliptisch):

Variation zwischen das und was

Eine elliptische Verwendung des Adjektivs liegt in Beispielen wie (33) vor, in denen das Adjektiv nicht substantiviert worden ist, sondern ein Substantiv modifiziert, das unter Identität mit einem diskursprominenten Element getilgt worden ist, vgl. (34).

Das bisher bestehende Kraftwerk ist bekanntlich völlig überaltet und muß schon allein aus Umweltgründen erneuert werden. Das neue, das Ende 1994 fertiggestellt sein soll, wird nach dem neuesten Stand der Technik errichtet und bis zu 90 Prozent weniger Schadstoffe an die Umwelt abgeben. (Salzburger Nachrichten, 5.3.1993; Heizkraftwerk Nord 88 Mill. S. teurer) ... Kraftwerk ... [Das neue Kraftwerk $\left[_{\text {SRel }}\right.$ das ...]] 
Elliptische Lesarten stellen also tatsächlich keinen relevanten Fall dar; in der Folge werden wir uns daher nur mit der nicht-elliptischen Verwendung von substantivierten Adjektiven befassen.

Wie bereits erwähnt, verhalten sich substantivierte Positive und Superlative nicht einheitlich. Auf der Basis einer Korpusstudie im DeREKo zeigt Fuß (i.Ersch.), dass substantivierte Superlative wie in (35) fast ausschließlich in Kombination mit was auftreten, vgl. Tabelle 1.

Abschließend gibt Angela Merkel ihrer Überzeugung Ausdruck, „dass der Blick von oben auf die Erde das Schönste ist, was es gibt“. (Berliner Zeitung, 21.7.2006, Ressort: Blickpunkt; „Wie klappt's mit dem Schlafen?", S. 3)

Tab. 1: das vs. was bei nicht-elliptischen Lesarten von substantivierten Superlativen

\begin{tabular}{lll}
\hline & das & was \\
\hline Beste(s) & $413(6,6 \%)$ & $5838(93,4 \%)$ \\
\hline Schönste(s) & $86(9,9 \%)$ & $783(\mathbf{9 0 , 1} \%)$ \\
\hline Neueste(s) & $11(\mathbf{1 6 , 9 \% )}$ & $54(\mathbf{8 3 , 1 \% )}$ \\
\hline
\end{tabular}

Auch hier liegt also nur wenig Variation vor (vgl. Brandt/Fuß 2015 zur Pragmatik von Fällen, in denen ausnahmsweise Relativierung mittels das vorliegt). Der Einfluss, den das Vorliegen einer superlativischen Form auf die Wahl des Relativums hat, lässt sich erklären, wenn man annimmt, dass sich die Bedeutung des Superlativs paraphrasieren lässt als ,mehr als alles (andere)‘, d.h., eine Kombination aus dem Komparativ und einer Bedeutungskomponente, die einen Allquantor als Vergleichsstandard enthält (vgl. z.B. Bobaljik 2012):

$$
\begin{aligned}
& \text { Superlativ = Komparativ }(\text {,mehr' })+\text {, als alles (andere) } \\
& \text { das Beste = ,etwas, das besser ist als alles (andere) }
\end{aligned}
$$

Vor diesem Hintergrund kann das Relativierungsverhalten von substantivierten Superlativen auf das Relativierungsverhalten von alles zurückgeführt werden (das ebenfalls fast ausschließlich was als Relativum auslöst, vgl. Abb. 5), wenn man annimmt, dass der Relativsatz den Vergleichsstandard ,als alles (andere)‘ modifiziert (Brandt/Fuß 2015):

$$
\text { das Beste, was es gibt = ,etwas, das besser ist als [alles, [was es gibt]] }
$$


Auf diese Weise ergibt sich eine weitere Einschränkung des variablen Bereichs, der nun nur noch das Relativierungsverhalten substantivierter nicht-gesteigerter Adjektive umfasst. Hier scheinen prinzipiell sowohl das als auch was als Relativum möglich zu sein, vgl. (39). Die Auswertung der Korpusbefunde zeigt allerdings, dass die $d$-Form überwiegt, vgl. Tabelle 2.

(39) a. In vielen Ländern Afrikas - wie auch Osteuropas - sind alte, überkommene Ordnungen oder Unordnungen im Umbruch oder sogar gestürzt. Das Neue, das sich aus solchen Veränderungen ergeben könnte, ist vielerorts allerdings noch nicht in Sicht. (die tageszeitung, 24.6.1992, S. 16; Zensur in der neuen Weltunordnung)

b. Aber auch ganz neue Ideen seien gerne willkommen und oft sei es das Neue, was den Anstoß gebe, einge fahrene Fahrwasser endlich zu verlassen. (Rhein-Zeitung, 5.7.1997; Gute Ideen sind in der Tat gefragt)

Tab. 2: das vs. was bei nicht-elliptischen Lesarten von substantivierten Positiven

\begin{tabular}{lll}
\hline & das & was \\
\hline Gute(s) & $490(74 \%)$ & $172(26 \%)$ \\
\hline Schöne(s) & $132(73,7 \%)$ & $47(26,3 \%)$ \\
\hline Neue(s) & $502(74,2 \%)$ & $175(25,8 \%)$ \\
\hline
\end{tabular}

Vor dem Hintergrund der Annahme, dass die Wahl zwischen das und was kategoriale Eigenschaften des Bezugselements reflektiert, kann das variable Relativierungsverhalten substantivierter Positive als Hinweis darauf verstanden werden, dass diese Elemente ,Mischkategorien' darstellen, die sowohl nominale als auch adjektivische Eigenschaften besitzen (vgl. Fuß i.Ersch. für eine ausführlichere Darstellung). Unabhängige Evidenz für einen solchen Analyseansatz ergibt sich z.B. aus der Beobachtung, dass substantivierte Positive zum einen die syntaktische Distribution von Nomina aufweisen (u.a. Präsenz eines Artikels), gleichzeitig aber auch typisch adjektivische Eigenschaften zeigen wie die Alternation zwischen starker und schwacher Flexion (das Gute vs. ein Gutes). Der spezielle kategoriale Charakter dieser Bildungen spiegelt sich in der Wahl des Relativums wider und führt zu (freier) Variation zwischen das und was. Die Tatsache, dass die Wahl des Relativums nicht eindeutig durch die Grammatik festgelegt ist, eröffnet zudem die Möglichkeit, dass die das/was-Alternation im Zusammenhang mit substantivierten Positiven zur Kodierung semantisch/pragmatischer Unterscheidungen genutzt werden kann. Generell gilt dabei, dass die Verwendung von 
das als Relativum eine individuierende Interpretation des Bezugselements befördert, während was verallgemeinernde Lesarten auslöst (vgl. Brandt/Fuß 2015 und Fuß i.Ersch. für eine nähere Auseinandersetzung).

\subsection{Außergrammatische Faktoren: Mündlichkeit vs. Schriftlichkeit}

Ein weiterer Faktor, der die Alternation zwischen das und was (stark) beeinflusst, ist außergrammatischer Natur und betrifft die Unterscheidung zwischen Mündlichkeit und Schriftlichkeit. Es ist ein bekanntes Phänomen, dass die Verwendung von was in mündlichen (v.a. umgangssprachlichen) Varietäten des Deutschen in Kombination mit Substantiven wesentlich gebräuchlicher ist (vgl. Murelli 2012). Obwohl DeReKo ausschließlich schriftliche Formate enthält, lässt sich dieser Effekt in einer Korpusstudie ebenfalls nachvollziehen. So kann man beobachten, dass in Belegen, die als direkte Rede gekennzeichnet sind (wie Interviews oder Zitate), sowie in Textsorten, die der Mündlichkeit nahe stehen (Parlamentsdebatten, Wikipedia-Diskussionen), was vermehrt auch in Konstruktion mit lexikalischen Nomina auftritt, vgl. (40) und Abbildung 6:

(40) Sein Trainer Dieter Hecking haderte mit der spielerischen Leistung: „Wir waren zu statisch in der 2. Halbzeit. Das 0:0 ist ein Ergebnis, was für uns nicht zufriedenstellend ist." (dpa, 22.8.2008; Magerkost in Hannover: 96 und Energie Cottbus trennen sich torlos)

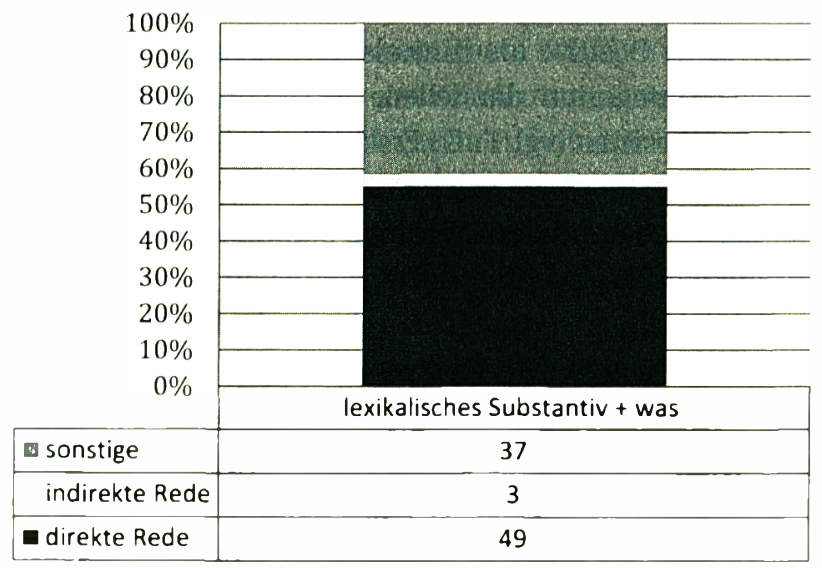

Abb. 8: Ergebnisse einer Stichprobe (600 aus 3.319 Belegen) für das Suchmuster ein/das N, was (DeReKo, Connexor Teilarchiv, 2.3.2016) 
Wie aus Abbildung 8 hervorgeht, lässt sich über die Hälfte aller Belege, in denen ein Substantiv durch einen was-Relativsatz modifiziert wird, als direkte Rede klassifizieren. Dies ist umso bemerkenswerter, als Zitate u.Ä. nur einen äußerst geringen Bruchteil aller Daten in DEREKo ausmachen.

\subsection{Zusammenfassung}

Bei der Untersuchung grammatischer Variation hat sich eine Strategie als vorteilhaft erwiesen, die eine schrittweise Reduktion der einschlägigen Fälle vornimmt, indem Faktoren ermittelt werden, welche die Distribution kombinatorischer Varianten regeln. Bei der vorliegenden Teilstudie haben wir zunächst starke grammatische Faktoren identifiziert, die die formale Variation zwischen das und was als Relativpronomina im Anschluss an Neutra im Singular steuern. Dabei haben wir für einen Beschreibungsansatz plädiert, der die Wahl zwischen $d$ - und $w$-Formen auf kategoriale Eigenschaften des Bezugselements zurückführt und im Gegensatz zu traditionellen Studien was als unterspezifiziertes Standard-Relativum betrachtet: Das spezifischere Relativum das steht nach Substantiven im Neutrum Singular, überall sonst steht was als Default-Form. Der Bereich, in dem ,echte Variation vorliegt, konnte auf das Relativierungsverhalten substantivierter Adjektive eingegrenzt werden. Ausgenommen hiervon sind allerdings substantivierte Superlative, die aufgrund ihrer spezifischen semantischen Eigenschaften in der Regel was als Relativum auslösen und somit einen weiteren starken Faktor darstellen. Der Kontext, in dem aus grammatischer Sicht tatsächlich freie Variation vorzuliegen scheint, beschränkt sich somit auf den relativischen Anschluss zu substantivierten Positiven (Neutrum Singular), der die letztlich verbleibende Variable darstellt (vorbehaltlich der Aufdeckung weiterer Faktoren). Das spezielle Verhalten dieser durch Konversion gebildeten Elemente haben wir darauf zurückgeführt, dass es sich hierbei um Mischkategorien (Nomen/Adjektiv) handelt, die mit beiden Einleitungstypen kompatibel sind. Es hat sich aber gezeigt, dass (vor allem in Fällen "freier" grammatischer Variation) die Verteilung der Varianten durch schwächere semantisch-pragmatische bzw. außergrammatische Faktoren beeinflusst wird, die ggf. noch näher zu untersuchen sind. So hat sich das als unterspezifiziertes Default-Relativum identifizierte was in mündlichen (v.a. umgangssprachlichen) Varietäten weiter ausgebreitet, wo es auch in Kombination mit Substantiven gebraucht werden kann. 


\section{Fazit}

Abschließend bleibt festzuhalten, dass die Untersuchung grammatischer Variation sowohl formale als auch funktionale Perspektiven zu berücksichtigen hat. Darüber hinaus müssen nicht nur grammatische, sondern auch außergrammatische bzw. sprachexterne Faktoren einbezogen werden. Dabei hat sich ein methodischer Ansatz bewährt, bei dem die relevante linguistische Variable anhand verschiedener Kontexte überprüft und ggf. immer weiter eingeschränkt wird, bis ausschließlich kombinatorische Varianten übrig bleiben bzw. sich ein Residuum freier Variation ergibt.

\section{Literatur}

Ammon, Ulrich (2005): Standard und Variation. Norm, Autorität, Legitimation. In: Eichinger, Ludwig M./Kallmeyer, Werner ( $\mathrm{Hg}$.): Standardvariation. Wie viel Variation verträgt die deutsche Sprache? (= Jahrbuch des Instituts für Deutsche Sprache 2004). Berlin/New York, S. $28-40$.

Ammon, Ulrich et al. (Hg.) (2008): Sociolinguistics. An international handbook of the science of language and society. 1. Halbbd. (= Handbücher zur Sprach- und Kommunikationswissenschaft 3.1). Berlin/New York.

Bausewein, Karin (1991): Acl-Konstruktionen und Valenz. In: Klein, Eberhard/Puradier Duteil, Francoise/Wagner, Karl Heinz (Hg.): Betriebslinguistik und Linguistikbetrieb. Bd. 1.

(= Linguistische Arbeiten 260). Tübingen, S. 245-251.

Bech, Gunnar (1955/1957): Studien über das deutsche verbum infinitum. Mit einem Vorwort von Catherine Fabricius-Hansen. Tübingen.

Behaghel, Otto (1928): Deutsche Syntax. Eine geschichtliche Darstellung. Bd. 3: Die Satzgebilde. Heidelberg.

Biber, Douglas/Jones, James K. (2009): Quantitative methods in corpus linguistics. In: Lüdeling, Anke/Kytö, Merja (Hg.): Corpus linguistics. An international handbook. 2. Halbbd. (= Handbücher zur Sprach- und Kommunikationswissenschaft 29.2). Berlin/New York, S. 1286-1304.

Bobaljik, Jonathan (2012): Universals in comparative morphology. Cambridge, MA.

Bortz, Jürgen/Dörig, Nicola (2006): Forschungsmethoden und Evaluation für Human- und Sozialwissenschaftler. 4. Aufl. Heidelberg.

Brandt, Patrick/Fuß, Eric (2014): Most questionable pronouns: Variation between das-vs. wasrelatives in German. In: Linguistische Berichte 239, S. 297-329.

Brandt, Patrick/Fuß, Eric (2015): Relativpronomenselektion und grammatische Variation: was vs. das in attributiven Relativsätzen. Ms., Institut für Deutsche Sprache. Mannheim.

Breindl, Eva/Volodina, Anna/Waßner, Ulrich Hermann (2014): Handbuch der deutschen Konnektoren 2. Semantik der deutschen Satzverknüpfer. 2 Teilbde. (= Schriften des Instituts für Deutsche Sprache 13). Berlin/Boston.

Bubenhofer, Noah/Konopka, Marek/Schneider, Roman (2014): Präliminarien einer Korpusgrammatik. (= Korpuslinguistik und interdisziplinäre Perspektiven auf Sprache 4). Tübingen. 
Chomsky, Noam/Lasnik, Howard (1977): Filters and control. In: Linguistic Inquiry 8, S. 425-504. Duden (2016): Der Duden in 12 Bänden. Bd. 4: Die Grammatik. Unentbehrlich für richtiges

Deutsch. 9., vollst. überarb. und aktual. Aufl. Berlin.

Durrell, Martin (2008): Linguistic variable - linguistic variant. In: Ammon et al. (Hg.), S. 195-200. Eisenberg, Peter (1994): Grundriß der deutschen Grammatik. 3., überarb. Aufl. Stuttgart. Eisenberg, Peter (2013): Grundriss der deutschen Grammatik. Bd. 2: Der Satz. 4.Aufl. Stuttgart. Felser, Claudia (1999): Verbal complement clauses: A minimalist study of direct perception constructions. Amsterdam/Philadelphia.

Fortmann, Christian (2013): Acl als Akkusativ plus Infinitiv-Adverbial. Ms. Tübingen.

Fuß, Eric (i.Ersch.): Relativierungsverhalten und syntaktische Kategorie substantivierter Adjektive. In: Döring, Sandra/Geilfuß-Wolfgang, Jochen (Hg.): Probleme der syntaktischen Kategorisierung: Einzelgänger, Außenseiter und mehr. Tübingen.

Haider, Hubert (1984): Was zu haben ist und was zu sein hat - Bemerkungen zum Infinitiv. In: Papiere zur Linguistik 30, S. 23-36.

Haider, Hubert (2010): The syntax of German. Cambridge.

Kiparsky, Paul (1973): ,Elsewhere' in phonology. In: Anderson, Stephen/Kiparsky, Paul (Hg.): A Festschrift for Morris Halle. New York, S. 93-106.

Kiparsky, Paul (1982): Word formation and the lexicon. In: Ingemann, Frances (Hg.): Proceedings of the 1982 Mid-America Linguistics Conference. Lawrence, S. 3-29.

Kutscher, Silvia (2014): Entwurf einer Makrostruktur zum Bereich der Sachverhaltsversprachlichung im Rahmen des Projekts Grammatik des Deutschen im europäischen Vergleich (GDE-V). (= Arbeitspapiere der Abteilung Grammatik 1). Mannheim. Internet: http:// ids-pub.bsz-bw.de/frontdoor/index/index/docld/3248 (Stand: 31.8.2016).

Labov, William (1966): The linguistic variable as a structural unit. In: Washington Linguistic Review 3, S. 4-22.

Labov, William (2008): Quantitative analysis of linguistic variation. In: Ammon et al. (Hg.), S. 6-21.

Lehmann, Christian (2004): Funktionale Grammatikographie. In: Premper, Waldfried (Hg.): Dimensionen und Kontinua. Bochum, S. 147-165.

Mittwoch, Anita (1990): On the distribution of bare infinitive complements in English. In: Journal of Linguistics 26, S. 103-131.

Murelli, Adriano (2012): Das Geheimnis, das oder was du mir verraten hast? - Das oder was als Relativpronomen. In: Konopka, Marek/Schneider, Roman (Hg.): Grammatische Stolpersteine digital. Festschrift für Bruno Strecker zum 65. Geburtstag. Mannheim, S. 145-152.

Reinhart, Tanja/Reuland, Eric (1993): Reflexivity. In: Linguistic Inquiry 24, S. 657-720.

Reis, Marga (1976): Reflexivierung in deutschen A.c.I.-Konstruktionen. Ein transformationsgrammatisches Dilemma. In: Papiere zur Linguistik 9, S. 5-82.

Reis, Marga (2001): Bilden Modalverben im Deutschen eine syntaktische Klasse? In: Müller, Reimar/Reis, Marga (Hg.): Modalität und Modalverben im Deutschen. (= Linguistische Berichte. Sonderheft 9). Hamburg, S. 287-318.

Rosenbaum, Peter S. (1967): The grammar of English predicate complement constructions. Cambridge, MA.

Schäfer, Roland/Bildhauer, Felix (2012): Building large corpora from the web using a new efficient tool chain. In: Calzolari, Nicoletta et al. ( $\mathrm{Hg}$.): Proceedings of the Eighth International Conference on Language Resources and Evaluation (LREC '12), Istanbul, May 21-25, 2012. Istanbul, S. 486-493. 
Speyer, Augustin (2015): Acl and control infinitives: How different are they? A diachronic approach. In: Journal of Historical Linguistics 5, S. 41-71.

Sternefeld Wolfgang (2006): Syntax. Eine morphologisch motivierte generative Beschreibung des Deutschen. (= Stauffenburg-Linguistik 31). Tübingen.

Thurmair, Maria (2001): Vergleiche und Vergleichen. Eine Studie zu Form und Funktion der Vergleichsstrukturen im Deutschen. (= Linguistische Arbeiten 433). Tübingen.

von der Gabelentz, Georg (1995): Die Sprachwissenschaft, ihre Aufgaben, Methoden und bisherigen Ergebnisse. In: Lepschy, Giulio C. ( Hg.): History of linguistics. 18th and 19th century German linguistics. Bd. 7. London/Tokyo. [Nachdruck der 2. verm. u. verb. Aufl., Leipzig 1901].

Wiese, Bernd (2013): Relativpronomina: Flexion und Wortfelder. Ms., Institut für Deutsche Sprache. Mannheim.

Zifonun, Gisela/Hoffmann, Ludger/Strecker, Bruno (1997): Grammatik der deutschen Sprache. 3 Bde. (= Schriften des Instituts für deutsche Sprache 7). Berlin/New York.

Zimmermann, Ilse (1991): Die subordinierende Konjunktion wie. In: Reis, Marga/Rosengren, Inger (Hg.): Fragesätze und Fragen. Referate anläßlich der 12. Jahrestagung der Deutschen Gesellschaft für Sprachwissenschaft, Saarbrücken 1990. (= Linguistische Arbeiten 257). Tübingen, S. 113-122. 